\title{
Backstepping based integral sliding mode control with neural network for ship steering control
}

\author{
Renqiang Wang ${ }^{a}$, Hua Deng, Keyin Miao, Yue Zhao, Jiabao Du \\ Navigation College, Jiangsu Maritime Institute, Nanjing 211170, PR China
}

\begin{abstract}
An integral sliding mode controller with neural network was investigated for ship navigation, which realizes the accurate and stable tracking of the ship in the steering process, on the basis of backstepping method. First of all, an integrator sliding surface were designed with the sliding mode variable structure control technology. Secondly, radial basis function neural network was applied to approximate the system nonlinear function and uncertain parameters. Furthermore, a nonlinear damping law was introduced to overcome the bounded outside interference. Finally, on the basis of the above, the system control law was deduced by using the backstepping method. The simulation results show that the neural network can accurately approximate the nonlinear function and uncertain parameters of the ship, and the controller output is smooth and the heading output is not sensitive to perturbation of parameters and external disturbance, and the controller has strong robustness.
\end{abstract}

\section{Introduction}

The factors that affect the performance of ship motion control are nonlinearity of ship motion system function, uncertainty of ship model parameters and unknown disturbance.

It is well known that the most advantage of sliding mode control [1] is able to overcome uncertainty of the system, which has highly robustness for uncertain dynamic system with outside disturbance, especially effectiveness for control of modelless system. It is an important method for suppressing high frequency switching that causes chattering of control input. And the variable structure control system algorithm is simple, fast response, and robustness to external noise and parameter perturbation. Therefore, it is useful for ship steering control. The integral link [2] joined into the algorithm of sliding mode control can also effectively eliminate the bounded interference outside so that the original controller has a stronger anti-jamming capability.

With the rapid development of computer technology, the performance of intelligent control [3], as the representative of neural network, has been improved greatly. The nonlinear function and the uncertain parameters can be arbitrarily identified by neural network with a universal approximation performance. The neural network [4] was used to infinitely approach and compensate the interference outside and the uncertainties of controlled system can reduce buffeting; The controller parameters were optimized online by genetic optimization method, so that the buffeting is reduced. A ship course RBF neural network sliding mode control algorithm is devised in literature [5], where sliding mode control based on the design of a hyperbolic tangent function for new reaching law can speed up the approaching speed and RBF neural network solves the problem of parameter variation and external disturbance. Global finite-time heading control of surface vehicles is designed in literature [6], which uses continuous control law to ensure the stability of the whole finite time closedloop control system and provide accurate ability of heading control with excellent anti-jamming capability. In literature [7], taking into consideration of the uncertainty of ship model parameter, a control algorithm based on RBF neurol network which approximates the uncertainty has been derived by using backstepping method.

Ship course response model has significant nonlinear characteristics, and backstepping [2] is a very effective method used to deal with the problem about nonlinear system controller design. It uses the system's structural properties, recursively constructs the entire system Lyapunov function, and makes the controller being more flexible and robust, but also reduces the difficulty and energy. Backstepping refers to the control system consists of a number of integrators in series, the state feedback controller is pushed through the integrator to the beginning of the control input, by way of coordinate transformation, systematic structure step by step and stabilization control Lyapunov function, so as to make the system asymptotically stable equilibrium point. Then, ship course sliding mode controller is designed in this paper by using backstepping method.

\footnotetext{
a Corresponding author: wangrenqiang2009@126.com
} 


\section{Problem description}

Considering the problem of ship maneuvering. the Bech [3] nonlinear model of ship motion nonlinear response was selected in paper, and its mathematical expression is:

$$
T_{1} T_{2} \ddot{\varphi}+\left(T_{1}+T_{2}\right) \ddot{\varphi}+H(\dot{\varphi})=K\left(T_{3} \dot{\delta}+\delta\right)+\omega_{0}
$$

Where, $T_{1}, T_{2}$ and $T_{3}$ represent ship follow index, $\mathrm{K}$ represents ship turning index, $\varphi$ represents ship course, $\delta$ represents rudder angle, $\omega_{0}$ represents ship external interference. And $H(\dot{\varphi})$ is nonlinear function with regard to $\dot{\varphi}$, which can be approximated as follows, $H(\dot{\varphi})=\alpha_{0}+\alpha_{1} \dot{\varphi}+\alpha_{2} \dot{\varphi}^{2}+\alpha_{3} \dot{\varphi}^{3}$.

Make the following settings:

$x_{1}=\varphi, x_{2}=\dot{x}_{1}=\dot{\varphi}=r, x_{3}=\dot{x}_{2}=\ddot{\varphi}=\dot{r}$, $a=\frac{1}{T_{1}}+\frac{1}{T_{2}}, b=\frac{1}{T_{1} T_{2}}, c=\frac{K}{T_{1} T_{2}}, u=T_{3} \dot{\delta}+\delta$.

Obviously, it is difficult to solve the system control input $\mathcal{U}$ which contains derivative items $T_{3} \dot{\delta}$, therefore, make the following setting $\omega=\omega_{0}+\frac{K}{T_{1} T_{2}} T_{3} \dot{\delta}$.

Therefore, formula (1) can be converted into the following format:

$$
\left\{\begin{array}{l}
\dot{x}_{1}=x_{2} \\
\dot{x}_{2}=x_{3} \\
\dot{x}_{3}=f(x)+c u+\omega \\
y=x_{1}
\end{array}\right.
$$

Where, $x_{1}, x_{2}$ and $x_{3}$ are system variable, $y$ is the actual output of the system, $\omega$ represents ship external interference, $u$ is the control input of system, $f(x)$ is system function, $f(x)=-\mathrm{b}\left(\alpha_{0}+\alpha_{1} x_{2}+\alpha_{2} x_{2}^{2}+\alpha_{3} x_{2}^{3}\right)-\mathrm{ax}_{3}$.

Based on above, the goal of this article is to seek control law $u$ which is able to make the system output $y$ asymptotically tracking expected course $\varphi_{r}$, the tracking error will be infinitely close to zero, that is $e=y-\varphi_{r} \rightarrow 0$, and the stabilization time will be reduced as much as possible.

\section{Dteering control design based on backstepping}

The design steps of the ship course controller contain the following steps.

(1) According to the characteristics of system (2), the following sliding surfaces are defined.

$$
\left\{\begin{array}{l}
\dot{\xi}=z_{3} \\
z_{1}=x_{1}-\varphi_{r} \\
z_{2}=x_{2}-\sigma_{1} \\
z_{3}=x_{3}-\sigma_{2}
\end{array}\right.
$$

Where, $\varphi_{r}$ is the expected course, $\sigma_{1}$ and $\sigma_{2}$ are virtual stabilization function, $Z_{1}$ is the heading error. $\xi$ is the integral term which can be able to eliminate the static error caused by the uncertain interference term in the control process.

(2) The second step, Lyapunov function is constructed to prove the asymptotic stability control system by using backstepping method. The first Lyapunov functionis constructed.

$$
V_{1}=\frac{1}{2} z_{1}^{2}
$$

According to (3), it is obtained (5).

$$
\dot{z}_{1}=z_{2}+\sigma_{1}-\dot{\varphi}_{r}
$$
(4).

It can be inferred formula (6) by derivation formula

$$
\dot{V}_{1}=z_{1} \dot{z}_{1}=z_{1}\left[z_{2}+\sigma_{1}-\dot{\varphi}_{r}\right]
$$

It can be obtained (7), according to (5) and (6).

$$
\dot{V}_{1}=-k_{1} z^{2}{ }_{1}+z_{1} z_{2}
$$

Based on the above, it can be obtained (8) by considering formula (5) and (7).

$$
\dot{z}_{1}=k_{1} z_{1}+z_{2}
$$

Therefore, it can be obtained (9).

$$
\dot{\sigma}_{1}=-k_{1}\left(-k_{1} z_{1}+z_{2}\right)+\ddot{\varphi}_{r}
$$

Similarly, according to (8), it is obtained (10).

$$
\dot{z}_{2}=\dot{x}_{2}-\dot{\sigma}_{1}=z_{3}+\sigma_{2}-\dot{\sigma}_{1}
$$

The second Lyapunov function is constructed.

$$
V_{2}=V_{1}+\frac{1}{2} z_{2}^{2}
$$

It can be inferred formula (12) by derivation formula (11).

$$
\begin{aligned}
\dot{V}_{2} & =\dot{V}_{1}+z_{2} \dot{z}_{2} \\
& =-k_{1} z_{1}^{2}+z_{2} z_{3}+z_{2}\left(z_{1}+\sigma_{2}-\dot{\sigma}_{1}\right)
\end{aligned}
$$

Making the following setting.

$$
\sigma_{2}=-k_{2} z_{2}-z_{1}+\dot{\sigma}_{1}
$$

It can be inferred formula (14) by (13) converted into (12).

$$
\dot{V}_{2}=-k_{1} z_{1}^{2}-k_{2} z_{2}^{2}+z_{2} z_{3}
$$

Therefore, it can be obtained (15) by considering formula (10) and (13).

$$
\dot{z}_{2}=-k_{2} z_{2}-z_{1}+z_{3}
$$

It is also acquired (16) by considering formula (13) and (15).

$$
\dot{\sigma}_{2}=\left(k_{1}^{2}-1\right) \dot{z}_{1}-\left(k_{1}+k_{2}\right) \dot{z}_{2}+\dddot{\varphi}_{r}
$$

And the third Lyapunov function is constructed. 


$$
V_{3}=V_{2}+\frac{1}{2} z_{3}^{2}+\frac{\lambda}{2} \xi^{2}
$$

It is easy obtained (18) by formula (3).

$$
\dot{z}_{3}=f(x)+c u+\omega-\dot{\sigma}_{2}
$$

It can be inferred formula (19) by derivation formula (17).

$$
\begin{aligned}
\dot{V}_{3}=\dot{V}_{2}+z_{3} \dot{z}_{3}+\lambda \xi z_{3} \\
=-k_{1} z^{2}{ }_{1}-k_{2} z_{2}^{2}+z_{3}\left(z_{2}+\dot{z}_{3}+\lambda \xi\right) \\
=-k_{1} z_{1}^{2}-k_{2} z_{2}^{2}+z_{2} z_{3}+\lambda \xi z_{3} \\
\quad+z_{3}\left[f(x)+c u+\omega-\dot{\sigma}_{2}\right]
\end{aligned}
$$

(3) The RBF network is used to adapt the system function $f(x)$, and its algorithm is (20).

$$
\left\{\begin{array}{l}
h_{j}=\exp \left(-\frac{\left\|x-c_{j}\right\|^{2}}{2 b_{j}^{2}}\right), j=1,2, \cdots, m \\
f(x)=W^{*^{T}} \mathbf{h}(x)+\varepsilon
\end{array}\right.
$$

Where $\boldsymbol{x}$ is the network input, $i$ represents one of the first input network input layer, $j$ is a hidden layer of the network the first network input, $\boldsymbol{h}=\left[h_{j}\right]^{\mathrm{T}}$ is high Gaussian function, $\boldsymbol{W}^{*}$ is the ideal for weighting value, $\varepsilon$ is ideal for neural network approximation error.

$$
\hat{f}\left(x_{2}\right)=\hat{W}^{T} \mathbf{h}(\mathbf{x})
$$

Where, $\tilde{W}$ is network connection weight adjustment error, there is $\tilde{W}=\hat{W}-W^{*}$.

Therefore, the compensation control law is described as formula (22).

$$
u=\frac{1}{c}\left[-\hat{f}(x)-\omega-\lambda \xi+\dot{\sigma}_{2}-z_{2}-k_{3} z_{3}\right]
$$

The global Lyapunov function is constructed.

$$
V=V_{3}+\frac{1}{2} \gamma \tilde{W}^{T} \tilde{W}
$$
(23).

It can be inferred formula (24) by derivation formula

$$
\dot{V}=\dot{V}_{3}+\gamma \tilde{W}^{T} \dot{\hat{W}}
$$

It can be obtained formula (25) by considering formula (19) and (22).

$$
\begin{aligned}
\dot{V}= & -k_{1} z_{1}^{2}-k_{2} z_{2}^{2}-k_{3} z_{3}^{2} \\
& +[f(x) \hat{-f}(x)] z_{3}+\gamma \tilde{W}^{T} \dot{\hat{W}} \\
= & -k_{1} z_{1}^{2}-k_{2} z_{2}^{2}-k_{3} z_{3}^{2}+\tilde{W}^{T}\left(\gamma \dot{\hat{W}}-\mathrm{h} z_{3}\right)
\end{aligned}
$$

In order to ensure system asymptotically stable, the following adaptive law is designed.

$$
\dot{\hat{W}}=\frac{1}{\gamma} \mathbf{h} z_{3}
$$

(4) In order to eliminate the uncertain interference term in equation (19), the nonlinear damping law [9] was designed.

$$
u=\frac{1}{c}\left[\begin{array}{c}
-f(x)-\lambda \xi+\dot{\sigma}_{2} \\
-z_{2}-\left(k_{3}+\eta\right) z_{3}
\end{array}\right]
$$

It can be inferred formula (28) by considering formula (25) and (27).

$$
\begin{aligned}
\dot{V}= & -k_{1} z_{1}^{2}-k_{2} z_{2}^{2}-k_{3} z_{3}^{2}-\eta z_{3}^{2} \\
& +(\omega+\varepsilon) z_{3}+\tilde{W}^{T}\left(\gamma \dot{\hat{W}}-\mathrm{h} z_{3}\right)
\end{aligned}
$$

Therefore, it is established that $V \leq 0$, and the system (2) is asymptotically stable.

It is easy obtained formula (29) by theorem " $x y \leq \eta x^{2}+\frac{1}{4 \eta} y^{2} "$.

$$
\varpi z_{3} \leq \eta z_{3}^{2}+\frac{1}{4 \eta}\|\varpi\|_{\infty}^{2}
$$

Where, $\varpi=\omega+\varepsilon$.

The fitness law of the network weights is still adopted formula (26), therefore, the formula (30) can be acquired.

$$
\begin{aligned}
\dot{V} & =-k_{1} z_{1}^{2}-k_{2} z_{2}^{2}-k_{3} z_{3}^{2}-\eta z_{3}^{2}+\varpi z_{3} \\
& \leq-k_{1} z_{1}^{2}-k_{2} z_{2}^{2}-k_{3} z_{3}^{2}+\frac{1}{4 \eta}\|\varpi\|_{\infty}^{2} \\
& \leq-k_{2} z_{2}^{2}+\frac{1}{4 \eta}\|\varpi\|_{\infty}^{2}
\end{aligned}
$$

In order to ensure that the system (4) is asymptotically stable, it is only need to prove the establishment which is $k_{2} z_{2}^{2} \geq \frac{1}{4 \eta}\|\omega\|_{\infty}^{2}$. Proof slightly, see the literature [8].

\section{Simulation}

Ship course continuous tracking control simulation is carried out by applying the above control algorithm.

\subsection{Simulation Object and Conditions Setting}

Effectiveness of control algorithm is verified through simulation of training vessel "YUKUN". It is known that the parameters of the ship are: $T_{1} \cdot T_{2}=9.238 \quad, \quad T_{1}+T_{2}=23.944 \quad, \quad K=7.926$, $T_{3}=10.064, \alpha_{0}=0, \alpha_{1}=1, \alpha_{2}=0, \alpha_{3}=30, a_{1}=1, a_{2}=30$, $K=0.2, T=64$.Outside interference is approximately substituted by white noise driven waves pope [9].

A required mathematical model which can represent ship's actual performance [10] is selected as following

$$
\begin{aligned}
& \phi_{m}(k+2)+0.1 \phi_{m}(k+1)+0.0025 \phi_{m}(k) \\
& =0.0025 \phi_{r}(k)
\end{aligned}
$$

Where, $\phi_{m}(k)$ represents the characterize performance of ship heading. $\phi_{r}(k)$ is a processed input signal, and $\phi_{r}(k)=(\operatorname{sign}(\sin (\pi k / 500))+1) \pi / 12$, whose range is from $0^{\circ}$ to $30^{\circ}$ and period is $500 \mathrm{~s}$.

\subsection{Simulation Results and Analysis}


Simulation was executed by Matlab/ Simulink, whose results were shown in figure 1 and figure 2.

Figure 1 depicts the curves of ship course and desired course, although the system parameter is changing, it rarely affects the tracking performances, which primarily affected by outside interference completely inhibited in the paper. Through the simulation demonstrated in Figure 1 , it can be concluded that the time complexity of the algorithm presented in the paper is substantially approximately linear order $O(n)$, and the proposed algorithm frequency time $T(n)$ is approximately $51 n$.

Figure 2 depicts the curves of control rudder angle, which is smooth and no buffeting and met the actual needs of the project.

Overall, the control system is stable, reliable, and which has strong robustness.

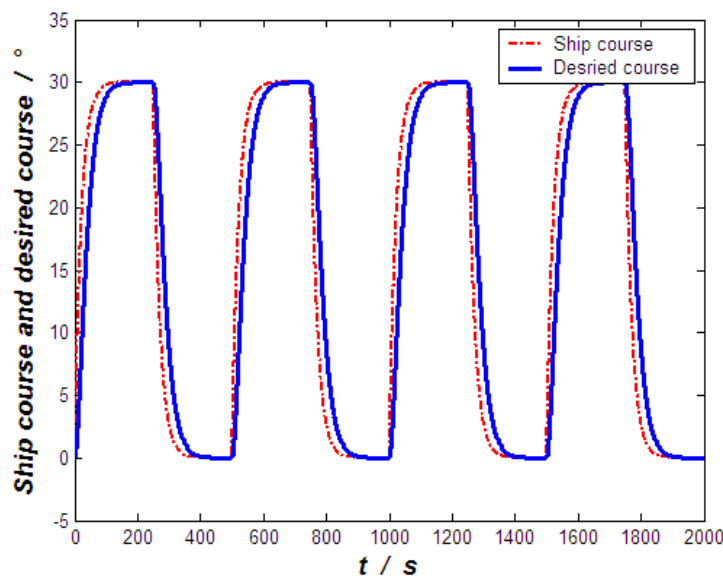

Fig.1. Curves of ship course and desired course

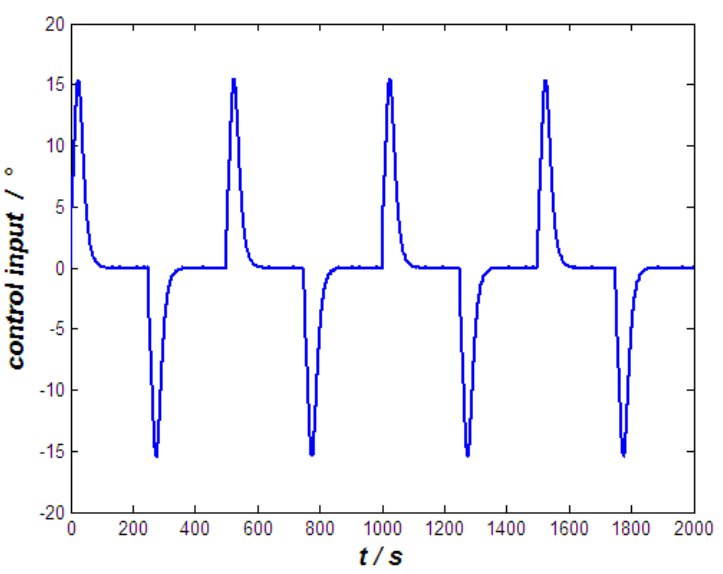

Fig.2. Curve of control rudder angle

\section{Conclusion}

A ship steering neural network controller based on integral sliding mode was designed by using the backstepping algorithm, based on the Bech ship motion mathematical model, for ship 's large scale maneuvering problem. The results show that the output of the controller is smooth and no jitter, and the heading output is not sensitive to the perturbation of the ship parameters and the external disturbance. Therefore, it has strong robustness.

\section{Acknowledgment}

This work was supported by the Foundation of Jiangsu Maritime Institute under Grant No.XR1501 and No.XR1504, and Jiangsu University Brand Professional Construction Project under Grant No. PPZY2015B177.

\section{References}

1. F. J Lin, W. D Chou, An Induction Motor Servo Drive using Sliding mode Control with Genetic Algorithem, Electric Power System Research, 2003, 64(2): 93-108.

2. Zhang Xianku.Ship motion simple and robust control, Beijing:Science Press, 2012.

3. Renqiang Wang, Yuelin Zhao, Keyin Miao, Application of Neural Network Minimum Parameter Learning Algorithm in Ship's Heading Tracking Control, Proceedings of 9th International Symposium on Computational Intelligence and Design, 2016, 12: 135-139.

4. Wang Renqiang, Zhao Yuelin, Sun Jianming, Application of Optimized RBF Neural Network in Ship's Autopilot Design, Proceedings of IEEE Advanced Information Management, Communicates, Electronic and Automation Control Conference, 2016, 10: 1642-1646.

5. Wang Renqiang, Gong Jianyun, Zhao Yuelin, Neural Network Sliding Mode Control under New Reaching Law and Application, Proceedings of Advanced Information Technology, Electronic and Automation Control Conference, 2015, 12: 911-917.

6. Wang Ning, Lv Shuailin, Liu Zhongzhong, Global finite-time heading control of surface vehicles, Neurocomputing, 2016, 175(106): 662-666.

7. Yang Wang, Chen Guo, Fuchun Sun. Dynamic neural fuzzified adaptive control of ship course with parametric modelling uncertainties, Int. J. of Modelling, Identification and Control, 2015, 13(4): 251-258.

8. Benaskeur A. R, Desbines A. Backstepping based adaptive PID control, Proceedings of Control Theory and Applications, 2002, 149(1): 54-59.

9. Guanshan $\mathrm{Hu}$, Yingbing Zhou, Application of Fuzzy Neural Network in Ship Course Control, Applied Mechanics and Materials, 2012, 1511(135): 309-315.

10. Xin Wang, Tieshan Li, Weilin Luo, Direct Adaptive Neural Network Control for a Class of Ship Course Uncertain Discrete-time Nonlinear Systems, Marine Engineering Frontiers, 2013, 37(1): 42-48. 